\title{
CURCUMIN PROTECTS THE SEMINAL VESICLES FROM METRONIDAZOLE-INDUCED REDUCTION OF SECRETION IN MICE
}

\begin{abstract}
Ali Noorafshan, Saied Karbalay-Doust
Histomorphometry \& Stereology Research Centre of Shiraz University of Medical Sciences, Shiraz, Iran

Summary: Seminal vesicle secretion is important for increasing the stability of sperm chromatin, inhibition of the immune activity in the female reproductive tract and so on. Metronidazole (MTZ), a drug used for treatment of infections caused by anaerobic bacteria and protozoa, may have negative effects on the genital gland including the seminal vesicles. Curcumin exhibits antioxidant as well as anti-inflammatory properties. The present study aims to evaluate the negative effects of MTZ on the seminal vesicle structure and ameliorative effects of curcumin using stereological methods. Thirty balb/c mice were divided into six groups. The control group was received distilled water. The second and the third received higher doses of MTZ (500 mg/kg body weight/day) and MTZ (500 mg/kg/day) $+100 \mathrm{mg} / \mathrm{kg} /$ day curcumin, respectively. The fourth and the fifth were treated with lower doses of MTZ (165 mg/kg body weight/day) and MTZ (165 mg/kg body weight/day) + curcumin $(100 \mathrm{mg} / \mathrm{kg}$ body weight/day), respectively. The sixth group received $100 \mathrm{mg} / \mathrm{kg}$ body weight/day curcumin. All the administrations were done by oral gavages for 14 days. After 30 days, seminal vesicles were removed. Stereological study of the seminal vesicle structure revealed a significant reduction in gland and vesicular fluid volume in MTZ-treated (higher or lower doses) animals. Curcumin protected the reduction of both parameters in therapeutic-dose treated animals. Metronidazole treatment does not induce structural changes in the seminal gland; however, it can have a significant impact on its secretion ability. Importantly, these deteriorations might be preventable by curcumin co-treatment.
\end{abstract}

Key words: Seminal vesicle; Metronidazole; Curcumin; Stereology

\section{Introduction}

Seminal vesicles are important accessory glands of most male mammals. They play a key role in male fertility process (10). It has been reported that seminal vesicle secretion is important for increasing stability of sperm chromatin, and inhibition of the immune activity in the female reproductive tract $(10,23)$. Furthermore, the sexual function of males is strongly related to the volume of seminal vesicles (10, 23). The abnormal function of the gland may give rise to sexual dysfunction and infertility in males $(10,23)$. The two seminal vesicles form approximately 60 percent of the fluids which pass from the male reproductive tract during ejaculation. In some mammals, the capacity of the seminal vesicles is much larger; the boar, for example, may increase the seminal fluid to 50 times as much. The most secretion include amino acids, citrate, enzymes, flavins, fructose (the main energy source of spermatocyte which relies entirely on sugars from the seminal plasma), phosphorylcholine, proteins, vitamin $\mathrm{C}$, and prostaglandins (which involves in suppressing an immune response by the female against the foreign semen) $(11,23)$.

Metronidazole is a nitroimidazole antibiotic medication and is used particularly for anaerobic bacteria and proto- zoa $(6,9,18)$. It has been demonstrated that metronidazole decreases the weight of the testis, epididymis, prostates, and also seminal vesicles after one month from the onset of the metronidazole treatment $(6,9,18)$. In addition, MTZ-treatment is related with reproductive hormone level changes $(12,27)$. However, study on the seminal vesicle structure - after the treatment with MTZ - by using stereological methods has received little attention. On the other hand, in the present study, ameliorative effect of curcumin on the seminal vesicle MTZ-treated mice was evaluated. Curcumin (diferuloylmethane) is the main curcuminoid of the turmeric which is a known spice. It has been shown that curcuminoids are polyphenols and are responsible for the yellow-orange color of the turmeric $(3-5,7,14$, 16). Many studies - including in vitro and animal studies - have suggested that curcumin may have antitumor, antioxidant, antiarthritic, anti-amyloid, anti-ischemic, and anti-inflammatory properties $(17,24,26,29)$.

Our previous study has showed the ameliorative effects of curcumin on the testis structure in MTZ-treated mice (21, 20). The aim of the present study is to evaluate the effects of higher $(500 \mathrm{mg} / \mathrm{kg} /$ day $)$ and lower doses $(165 \mathrm{mg} / \mathrm{kg} /$ day $)$ of MTZ on the seminal vesicle. It also aims to show the effects of curcumin on the seminal vesicle after the treatment. To 
have a quantitative and comparable data about the seminal vesicle structure after the MTZ and the curcumin treatment, stereological methods are used. Volume of the seminal vesicle, its parenchyma, vesicular fluid, epithelial cells, vessels, and connective as well as muscular tissues are evaluated.

\section{Materials and Methods}

Ethics. All animal experiments were approved by the Animal Ethics Committee of Shiraz University of Medical Sciences.

Animals. 30 balb-c mice weighing between 35-40 grams were selected from the Laboratory Animal House of Shiraz University of Medical Sciences. The animals were housed in the standard condition with free access to food and water. The mice were acclimatized for two weeks and were divided into six groups each including 5 mice. The control group received distilled water. The second group received higher doses of metronidazole (500 mg/kg body weight/day) $(6,9$, $18)$. The third group, also, received higher doses of metronidazole (500 mg/kg body weight/day) plus $100 \mathrm{mg} / \mathrm{kg} /$ day curcumin (26). The forth, on the other hand received lower doses of metronidazole (165 mg/kg body weight/day) $(6,9$, 18). The fifth group, also, received lower doses of metronidazole (165 mg/kg/ body weight/day) plus $100 \mathrm{mg} / \mathrm{kg} /$ day curcumin. Finally, the sixth group received $100 \mathrm{mg} / \mathrm{kg}$ body weight/day curcumin. All the administrations were done by oral gavages for 14 days.

\section{Stereological study}

After 30 days, the animals were weighed and anaesthetized. Bilaterally situated seminal vesicles, excluding the ampula of vas deference, ejaculatory duct, and the connective tissue, wer dissected out and weighed.

The primary volume - "V $\mathrm{V}_{\text {primary }}$ " - was measured using the immersion method (25). A brief explanation can be found under Figure 1. The final volume of the seminal vesicle should be estimated in the stereological study in order to prevent the reference trap $(2,22,19)$. Different methods such as Cavalieri methods can be used for estimating the seminal vesicle volume, but this technique needs consecutive sections and it is time consuming. In this study, the final gland volume was estimated after shrinkage estimation without the need to consecutive sections. Tissue shrinkage is mainly due to the tissue processing and staining. Estimation of some stereological parameters, such as shrinkage, requires isotropic uniform random sections (i.e. the gland has a random position and orientation during sectioning and all its parts have an equal chance to be sectioned) $(19,22)$. These sections were obtained by the "orientator" method (Figure 2). Briefly, each lobe of the gland was placed on a circle divided into 10 equal distances. A random number between 0 and 10 was selected and the gland was sectioned into two halves using a blade at that direction. The cut surface of each half of the lobe was then placed on the $0-0$ direction of the second circle with 10 un- equal cosine-weighted divisions and, after that, the second cut was done. Each half was sectioned into slabs with a blade, and was placed then in the direction of the second cut. Then, the 8-10 slabs were collected. A circle was punched from a seminal vesicle slab by a trocar (Figure 2). Two random diameters of the circular piece of the gland were measured by a micrometer. Besides, the area of the circle was estimated using the usual formula for calculating the area of a circle. The cut surfaces of the slabs and the circular piece were embedded in paraffin block and only one section with $5 \mu \mathrm{m}$ thicknesses was obtained. The section was stained with Heidenhain's AZAN trichrome stain. After staining, the area of the circular piece

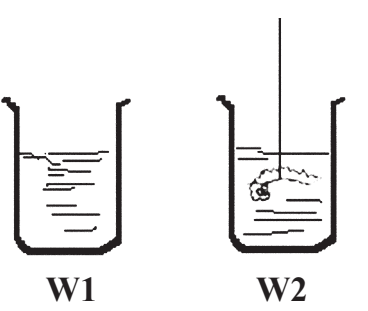

Fig. 1: Immersion method to estimate the volume of the seminal vesicle. A container with distilled water was placed on the scale and weighed (W1). Then, the seminal vesicle suspended by a thin thread was immersed in the second container in a way that it was fully covered by water while did not touch the bottom of the container. The new weight in grams was recorded (W2). This weight minus the weight of the container and the water was divided by the specific gravity of the distilled water $(\sim 1.0)$ was the volume of the gland in cubic centimeters.

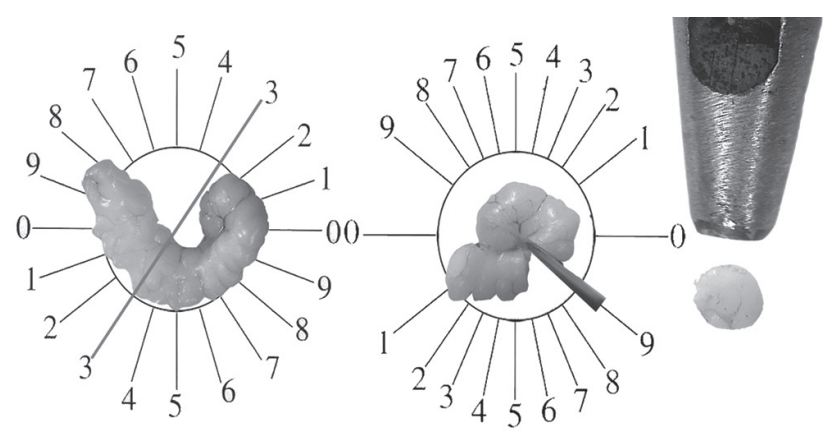

Fig. 2: Orientator method. Left. Each lobe of the seminl vesicle was placed on a circle divided into 10 equal distances. A random number between 0 and 10 was selected and the gland was sectioned into two halves using a blade at that direction (here 3). Middle. The cut surface of each half of the lobe was then placed on the second circle with 10 unequal cosine-weighted divisions and the second cut was done at a random direction (here 9). Right. A circle was punched from a seminal vesicle slab by a trocar. 
was measured again and the volume shrinkage $(19,22)$ was calculated using the following formula:

$$
\text { Volume shrinkage }=1-(\mathrm{AA} / \mathrm{AB})^{1.5}
$$

where "AA" and "AB" were the area of the circular piece after and before the processing, sectioning, and staining, respectively.

After estimating the shrinkage, the final volume of the gland (the reference space) was estimated using the following formula:

$$
\mathrm{V}_{\text {final seminal vesicle }}=\mathrm{V}_{\text {primary }} \times(1-\text { volume shrinkage }) .
$$

Each sampled section was analyzed using a video-microscopy system which consisted of a microscope (E-200, Nikon, Japan) which was linked to a video camera (Sony, Japan, SSC Dc 18P), a computer, and a flat monitor. On the first step of the estimation the sections were evaluated at final magnification of $\times 10$ and the volume fraction of the glandular parenchyma and secretion were estimated. The fraction was estimated using the point-counting method (Figure 3). A probe which included points was laid on the microscopic images by means of the stereology software designed at our laboratory (Morphometry \& Stereology Research Laboratory, Shiraz University of Medical Sciences, Shiraz, Iran). The following formula was applied:

$$
\left.\mathrm{Vv}_{(\text {structure/gland }}\right)=\mathrm{P}_{\text {(structure) }} / \mathrm{P}_{\text {(gland) }} \text {, }
$$

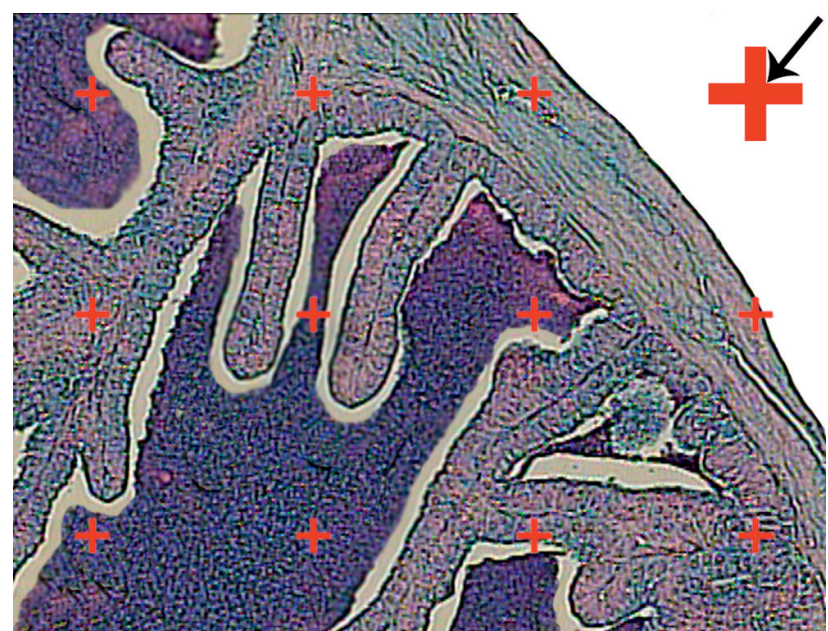

Fig. 3: Point counting method. A probe which consisted of points was laid on the microscopic images by means of the stereology software. In order to achieve the volume fraction of each structure, the number of points which hit the profiles of the epithelial cells, vessels, connective tissue, muscular layer, or vesicular fluid was counted and was divided by the total number of points hitting the whole seminal vesicle sections. The magnified cross shows the definition of a point which is the right upper corner of the cross. If only the right upper corner of the cross was laid on each parameter it was counted. where the $\mathrm{P}_{\text {(structure) }}$ and $\mathrm{P}_{\text {(gland) }}$ were the number of points hitting the profiles of the parenchyma or vesicular fluid and the whole seminal vesicle sections, respectively. The volume fraction was multiplied by the final volume of the seminal vesicle.

To estimate volume fraction of the constituents of the parenchyma, 10-14 microscopic fields were examined in each gland at final magnification of $1800 \times$. To select the microscopic fields, a systematic random sampling procedure was applied, i.e. the position of the first field was selected randomly outside the sections. Then, the slide was moved at equal intervals along the $\mathrm{X}$ - and $\mathrm{Y}$-axis using a stage micrometer until all the sections on one slide had been studied. The point counting method and the following formula were used:

$$
\mathrm{VV}_{\text {(structure/ parenchyma) }}=\mathrm{P}_{\text {(structure) }} / \mathrm{P}_{\text {(parenchyma) }},
$$

where the $\mathrm{P}_{\text {(structure) }}$ was the number of the points which hit the profiles of the epithelial cells, vessels, connective, and smooth muscle tissues. The volume fraction of each parameter was multiplied by the absolute volume of the parenchyma.

\section{Statistical Analysis}

Statistical comparisons between the group means were done by Mann-Whitney U-test and Kruskal-Wallis. P-values less than 0.05 were considered as significant.

\section{Results}

After the lower and higher doses of MTZ treatment, the seminal gland volume decreased $26 \%$ and $33 \%$, respectively, in comparison to the control group $(\mathrm{p}<0.04)$. Moreover, after the treatment with lower and higher doses of $\mathrm{M} \mathrm{TZ}$, the vesicular fluid volume decreased $33 \%$ and $40 \%$, respectively, in comparison to the control group $(\mathrm{p}<0.04)$. The reduction in the seminal gland and the vesicular fluid volume remained the same in lower dose MTZ-treated animals after the curcumin treatment. Volumes of the parenchyma, epithelial cells, vessels, and connective as well as muscular tissues did not show any significant changes. The results showed that MTZ did not have any significant influence on the gland structure, but the secretion ability was diminished. It should be noted that curcumin failed to protect the seminal vesicles from toxicity of the higher dose of metronidazol. The data is presented in the Table 1.

\section{Discussion}

The present study evaluated the effects of different doses of metronidazole with or without curcumin treatment on the seminal gland of mice. The importance of the accessory sex organs on fertility in mice was confirmed in different studies 
Tab. 1: The mean \pm the standard deviation of the weight $(\mathrm{mg})$, volume $\left(\mathrm{mm}^{3}\right)$ of the seminal vesicle (SV), parenchyma (PCH), vesicular fluid (FLUID), epithelial cells (CELL), vessels (VES), connective (CT) and muscular tissue (MT) in higher (500) and lower (165) doses of metronidazole (MTZ) and/or curcumin (CUR) treated groups.

\begin{tabular}{|l|l|l|l|l|l|l|l|l|}
\hline & Weight & \multicolumn{7}{|c|}{ Volume } \\
\hline Groups & SV & \multicolumn{1}{|c|}{ SV } & PCH & FLUID & CELL & VES & CT & MT \\
\hline Control & $210 \pm 44^{*}$ & $172 \pm 36^{*}$ & $39.5 \pm 13$ & $130.9 \pm 30.9^{*}$ & $32.2 \pm 19.2$ & $0.07 \pm 0.06$ & $0.23 \pm 0.2$ & $12.4 \pm 1.2$ \\
\hline MTZ 500 & $140 \pm 23$ & $114 \pm 19$ & $35.2 \pm 11$ & $78.3 \pm 12.6$ & $25.7 \pm 12.6$ & $0.10 \pm 0.08$ & $0.51 \pm 0.5$ & $8.2 \pm 1.4$ \\
\hline $\begin{array}{l}\text { MTZ 500 } \\
+ \text { CUR }\end{array}$ & $144 \pm 11$ & $118 \pm 9$ & $37.9 \pm 6$ & $81.6 \pm 13.5$ & $31.6 \pm 4.8$ & $0.04 \pm 0.01$ & $0.68 \pm 0.1$ & $5.6 \pm 1.6$ \\
\hline MTZ 165 & $154 \pm 27$ & $126 \pm 23$ & $39.3 \pm 9$ & $86.6 \pm 25.9$ & $33.8 \pm 9.6$ & $0.04 \pm 0.01$ & $0.12 \pm 0.1$ & $8.1 \pm 1.7$ \\
\hline $\begin{array}{l}\text { MTZ 165 } \\
+ \text { CUR }\end{array}$ & $210 \pm 25^{* *}$ & $172 \pm 20^{* *}$ & $47.4 \pm 9$ & $123.0 \pm 25.0 * *$ & $32.8 \pm 5.9$ & $0.11 \pm 0.08$ & $0.62 \pm 0.3$ & $13.8 \pm 1.7$ \\
\hline CUR & $172 \pm 31$ & $141 \pm 26$ & $39.4 \pm 13$ & $99.7 \pm 37.0$ & $25.6 \pm 16.0$ & $0.03 \pm 0.01$ & $0.88 \pm 0.6$ & $12.8 \pm 1.3$ \\
\hline
\end{tabular}

$* \mathrm{p}<0.046$, Control vs. (MTZ 500 and MTZ 165)

** $\mathrm{p}<0.021$, (MTZ $165+$ CUR) vs. MTZ 165

including those of Pang et al. (1979) and Gonzales (2001) $(23,10)$. Pang and colleagues in an interesting study showed the birth out come of the female mice which were mated with the males without the accessory genital glands. The results showed that, although both coagulating glands and seminal vesicles are necessary for the production of a vaginal plug in rodents, the removal of the seminal vesicles had a much greater effect on fertility than did the removal of the coagulating glands. In the present research, the importance of the seminal vesicle was evaluated after the metronidazole treatment. The reduction of the weight of the gland after the MTZ-treatment has been reported $(6,18)$. This is in accordance with our findings. In addition, in order to show which part of the gland has been responsible for the weight and the volume reduction, the present stereological methods were applied. Our study showed that the secretion of the gland has been reduced after the MTZ-treatment, while no structural changes have been seen. Thus, it seems that MTZ can disturb the secretary function of the gland. The underlying mechanism for this disturbances might be some reductions in reproductive hormones such as: the follicle stimulating hormone, the luteinizing hormone, and testosterone. Grover et al. (2001) and Sohrabi et al. (2007) have reported that significant reductions in the mean serum FSH, $\mathrm{LH}$, and testosterone level were seen in metronidazole treated animals $(12,27)$. It has been reported that the growth of the seminal vesicles are highly dependent on androgens (13-15). An experiment has shown that the endogenous increase of the serum testosterone level increased the secretary activity of the seminal vesicles in men (11). Other studies reported that any increase in the serum testosterone or treatment with androgens was associated with the increased secretary activity and the weight of the seminal vesicles in rats $(30,8,1,28)$. In addition, it is possible that MTZ may decrease the production of the sexual hormones via its direct effect or the toxic damage. On the other hand, curcumin can ameliorate the induced injuries of the reproductive glands. The ameliorate effects of curcumin against the testicular damage by the di-n-butylphthalate, cisplatin, aflatoxin, and ischemia-reperfusion has been documented $(5,7,12,16,17,26,29)$. Ilbey et al. (2009) showed that cisplatine decreases the plasma testosterone levels of rats; however, they observed a significant increase in the plasma testosterone levels after the treatment with curcumin. Thus, the protective effects of curcumin which were observed in the present study might be due to its ability to increase some reproductive hormones such as testosterone. This might be investigated in further studies.

\section{Conclusion}

The secretion fluid in seminal vesicle of mice can be decreased by both lower and higher doses of metronidazole. However, curcumin can only prevent lower doses of metronidazole from the decreasing the secretion fluid in the seminal vesicle of mice.

\section{Acknowledgements}

The work was carried out and financially supported by Histomorphometry \& Stereology Research center of Shiraz University of Medical Sciences. Miss. Elham Nadimi is acknowledged for her technical assistant.

\section{References}

1. Almenara A, Escalante G, Gazzo E, Gonzales GF. Transillumination to evaluate spermatogenesis: Effect of testosterone enanthate in adult male rats. Arch Androl 2000; 46(1): 21-7.

2. Braendgaard H, Gundersen HJ. The impact of recent stereological advances on quantitative studies of the nervous system. J Neurosci Methods 1986; 18(1-2): 39-78.

3. Cohly HH, Taylor A, Angel MF, Salahudeen AK. Effect of turmeric, turmerin and curcumin on $\mathrm{H}_{2} \mathrm{O}_{2}$-induced renal epithelial (LLC-PK1) cell injury. Free Radic Biol Med 1998; 24(1): 49-54.

4. Duvoix A, Blasius R, Delhalle S, et al. Chemopreventive and therapeutic effects of curcumin. Cancer Lett 2005; 223(2): 181-90.

5. El-Demerdash FM, Yousef MI, Radwan FM. Ameliorating effect of curcumin on sodium arsenite-induced oxidative damage and lipid peroxidation in different rat organs. Food Chem Toxicol 2009; 47(1): 249-54.

6. el-Nahas AF, el-Ashmawy IM. Reproductive and cytogenetic toxicity of metronidazole in male mice. Basic Clin Pharmacol Toxicol 2004; 94(5): 226-31. 
7. Farombi EO, Abarikwu SO, Adedara IA. Oyeyemi MO. Curcumin and kolaviron ameliorate di-n-butylphthalate-induced testicular damage in rats. Basic Clin Pharmacol Toxicol 2007; 100(1): 43-8.

8. Fawell SE, Higgins SJ, Androgen regulation of specific mRNAs, endoplasmic reticulum and Golgi-system. Mol Cell Endocrinol 1984; 37(1): 15-27.

9. Freeman CD, Klutman NE, Lamp KC. Metronidazole. A therapeutic review and update. Drugs 1997; 54(5): 679-708.

10. Gonzales GF. Function of seminal vesicles and their role on male fertility. Asian J Androl 2001; 3(4): 251-258.

11. Gonzales GF. Test for androgen activity at the male reproductive tract in infertile men. Arch Androl 1994; 32(3): 235-42.

12. Grover JK, Vats V, Srinivas M, et al. Effect of metronidazole on spermatogenesi and FSH, LH and testosterone levels of pre-pubertal rats. Indian J Exp Biol. 2001; 39(11): 1160-2

13. Higgins SJ, Burchell JM. Effects of testosterone on messenger ribonucleic acid and protein synthesis in rat seminal vesicle. Biochem J 1978; 174: 543-51.

14. Ilbey YO, Ozbek E, Cekmen M, Simsek A, Otunctemur A, Somay A. Protective effect of curcumin in cisplatin-induced oxidative injury in rat testis: mitogen-activated protein kinase and nuclear factor-kappa B signaling pathways. Hum Reprod 2009; 24(7): 1717-25.

15. Lieber MM, Barham SS, Veneziale CM. In vitro propagation of seminal vesicle epithelial cells. Invest Urol 1980; 17(4): 348-55.

16. Mathuria N, Verma RJ. Curcumin ameliorates aflatoxin-induced lipid peroxidation in liver, kidney and testis of mice - an in vitro study. Acta Pol Pharm 2007; 64(5): 413-6.

17. Mathuria N, Verma RJ. Curcumin ameliorates aflatoxin-induced toxicity in mice spermatozoa. Fertil Steril 2008; 90 (3): 775-80.

18. McClain RM, Downing JC, Edgcomb JE. Effect of metronidazole on fertility and testicular function in male rats. Fundam Appl Toxicol 1989; 12(3): 386-96.

19. Mühlfeld C, Nyengaard JR, Mayhew TM. A review of state-of-the-art stereology for better quantitative 3D morphology in cardiac research. Cardiovasc Pathol. 2010; 19(2): 65-82.

20. Noorafshan A, Karbalay-Doust S, Valizadeh A, Aliabadi E. Ameliorative effects of curcumin on the structural parameters of seminiferous tubules and Leydig cells in metronidazole-treated mice: A stereological approach. Exp Toxicol Pathol 2010 (Epub ahead of print).

21. Noorafshan A, Karbalay-Doust S, Valizadeh A, Aliabadi E, Mirkhani H. Ameliorative effects of curcumin on the seminiferous epithelium in metronidazole-treated mice: a stereological study. Toxicol Pathol 2010; 38(3): 366-71.

22. Nyengaard JR. Stereologic methods and their application in kidney research. J Am Soc Nephrol 1996; 10(5): 1100-23.

23. Pang SF, Chow PH, Wong TM. The role of the seminal vesicles, coagulating glands and prostate glands on the fertility and fecundity of mice. J Reprod Fert 1979; 56(1): 129-132.

24. Sahoo DK, Roy A, Chainy GB. Protective effects of vitamin E and curcumin on L-thyroxine-induced rat testicular oxidative stress. Chem Biol Interact 2008; 176(2-3): 121-8

25. Scherle W. A simple method for volumetry of organs in quantitative Stereology. Mikroskopie 1970; 26(1): 57-60.

26. Shu JC, He YJ, Lv X, Ye GR, Wang LX. Curcumin prevents liver fibrosis by inducing apoptosis and suppressing activation of hepatic stellate cells. J Nat Med 2009; 63(4): 415-20.

27. Sohrabi D, Alipour M, AwsatMellati A. Effect of Metronidazole on Spermatogenesis, Plasma Gonadotrophins and Testosterone in Male Rats. Iranian Journal of Pharmaceutical Research 2007; 6(4): 279-283.

28. Tao YX, Lei ZM, Rao CV. Seminal vesicles are novel sites of luteinizing hormone human chorionic gonadotropin receptor gene expression. J Androl 1998; 19(3): $343-7$.

29. Wei SM, Yan ZZ, Zhou J. Curcumin attenuates ischemia-reperfusion injury in rat testis. Fertil Steril 2009; 91(1): 271-7.

30. Zanato VF, Martins MP, Anselmo-Franci JA, Petenusci SO, LamanoCarvalho TL. Sexual development of male Wistar rats. Braz J Med Biol Res 1994; 27(5): $1273-80$

Received: $21 / 04 / 2011$

Accepted in revised form: 18/02/2012

\section{Corresponding author:}

Saied Karbalay-Doust, Histomorphometry \& Stereology Research Centre of Shiraz University of Medical Sciences, Shiraz, Iran; e-mail: karbalas@sums.ac.ir 